\title{
The design and implementation of a web-based teaching platform for folk sport courses in regular universities ${ }^{*}$
}

\author{
Qin WANG, Peijun GAO \\ Jiujiang University, Jiujiang, Jiangxi, 332005
}

\begin{abstract}
----with the rapid development of economy, the past decades have witnessed the booming of information technology, communication technology and multimedia technology, which have contributed to a plenty of new teaching modes in China's highe $\mathbf{r}$ education, such as distant education and web-based education. It is commonly recognized that folk sports courses embrace highly practical and participatory teaching activities, in which multimedia teaching activities are feasible and available. However, online teaching resources of the kind are still rare, incomplete and not yet systemized. This paper focuses on how to better design a shared web-based teaching platform for folk sports courses in regular universities and standardize online teaching resources, so as to realize the sustainable development of folk sports culture.
\end{abstract}

Keyword----folk sports; a web-based teaching platform; design and implementation

\section{普通高校民俗体育网络教学平台的设计与实现 ${ }^{*}$}

\author{
王勤 高培军 \\ (九江学院体育学院, 九江, 江西, 332005)
}

\begin{abstract}
摘 要: 如今经济的快速发展, 计算机技术、通信技术、和多媒体技术也得到了快速的发展, 它们的发展使我国的教育领域随之出 现了许多以教育技术为代表的新的教育模式, 其中远程教育和网络教育就是相当突出的一部分。而我们都知道民俗体育是一项参与 性与实践性极强的教与学的双边活动, 可惜的是目前网络上的教学资源相对比其他学科来说都要少, 而且也缺少相应的完整性和系 统性。所以本文重点探讨如何更好地设计我国普通高校民俗体育网络教学平台, 并网共享, 实现网络教学课件规范化、通用性, 从 而实现民俗体育文化可持续发展。
\end{abstract}

关键词: 民俗体育; 网络平台; 设计; 实现

1. 前言

普通高校通过开展民俗体育网络教学可以更好的利用 当前已经具备的各种体育教学资源, 使我们有限的体育器 材和体育场地以及师资力量等教育资源得到充分利用, 充 分提高普通高校体育课堂的教学质量, 丰富普通高校学生 的课外体育活动, 提高他们学习体育的积极性和主动性, 从而提高普通高校学生的身体素质。并且更有利于突破我 国那种传统的体育教学的时空限制, 以一种全新的方式更
好地促进普通高校学生课堂内外的体育学习, 为他们提供 更多新的学习时间和空间, 从而增强对他们的体育理论知 识的普及, 促进普通高校体育教学质量的提高。

由于实现民俗体育网络教学的方式多种多样, 而根据 体育学科的特点, 一般都采用基于 web 的教学系统、基于 点播以及实时交互的教学系统, 充分分析民俗体育网络教 学平台开发的需求, 从而充分利用相关的 WEB 技术、数据 库、流媒体技术以及电子白板等关键技术, 更好地地发展

* 致谢: 2014 年江西省高校教改项目（JXJG-14-17-4）的阶段成果之一。

作者简介: 王勤 (1963-) 男, 江西九江人, 九江学院副教授。主要从事体育教学及体育人文研究。 
民俗体育网络教学, 在标准的民俗体育网络教学平台设计 的准则下, 探讨对体育学科的网络教育平台的设计方法, 提出了总体的功能模块模型和结构模型, 促进江西省普通 高校民俗体育网络教学的发展, 更促进我国所有省份普通 高校民俗体育网络教学的发展。

\section{2. 民俗体育网络课件设计与实现的必要性}

体育教学是一项参与性与实践性极强的教与学的双边 活动, 普通高校开展体育远程网络教育可以更好的利用各 种体育教学资源, 使各种有限的体育器材体等教育资源得 到充分利用, 有利于提高体育课堂的教学质量, 丰富普通 高校学生的课外体育活动, 并且更有利于突破传统体育教 育的时空限制, 用一种全新的形式去促进学生课堂内外的 体育课程学习, 为普通高校大学生提供新的学习空间, 从 而有利于大大提高普通高校学生学习体育课的积极性和主 动性, 也有利于增强体育理论知识的普及, 从而更好的提 升普通高校学生的体育素质, 为我国社会主义事业培养更 优秀的接班人。

\section{3. 高校民俗体育网络教学平台设计与实现的现状与 困难}

总体上说来, 网络教育在我国或是国外发展的势头都 比较良好, 在各个普通高校或教学单位培训中的应用也越 来越广泛。我们都知道现在很多高校依托便利的校园网优 势, 开展对学科的网络教育。江西省普通高校民俗体育网 络教学平台的设计仍存在许多不足之处。虽然部分具备网 络学习的常用功能, 但是仍然有很多只是局限于课程内容 的展示, 对流媒体技术的应用和实时互交等空间并没有完 全发掘出来, 重点是缺乏相应的设计人才, 并且技术也不 到位, 国家的扶持力度也不是很大, 并没有相对健全的相 应的法规。

对于民俗体育教学领域来说, 可以较好地利用图形、 视频图像、动画、声音和文字等对民俗体育运动知识和运 动技能进行多角、多层次以及全方位的呈现, 使民俗体育 课程中相关的动作示范更真实更生动更贴近普通高校的学 生。由于现在民俗体育网络教育大多数都是理论研究, 而 真正有关民俗体育网络教育平台设计和平台开发方面的研 究和成果都是非常有限, 所以对于民俗体育网路教育这项 规模宏大的系统工程, 还需要我们去探索这其中许多未知 的领域, 促进我国普通高校民俗体育网络教学平台的设计 和发展。

\section{4. 如何更好地实现普通高校民俗体育网络教学平台 的设计}

普通高校民俗体育网络教学平台设计可以根据体育教 学特点, 在术科教学和遵循体育理论规律和原则的同时, 应体现出体育教学资源的广泛性和丰富性, 为民俗体育技 术动作的学习提供更多有利的指导, 也提供更多有效的学 习技巧和支持, 努力为学生审美意识的提高和各项能力的 发展创造有利的条件。要善于借助各种网络的学习环境, 使民俗体育网络课程教学设计符合网络环境教学的特点, 在进行网络教学设计时也要考虑到网络对教师和学生本身 的要求, 从而充分发挥网络的优势, 并关注网络的不足和 其自身的弱势, 在传统体育教学领域里寻找合理的弥补办 法。

4.1 为了教学的生动性和直观性, 普通高校民俗体育 要擅于使用各种大量的视频、动画、图片和文本等多媒体 素材。如: 图片类素材可以采用 JPEG、PNG、GIF 等格式, 预计 100 张左右即可; 而有关民俗体育动画类素材可以采 用 GIF、SWF 格式, 预计 20 张左右即可; 视频类素材我们 可以采用流媒体格式, 使用 MPEC、WMV、FLV 和 RM 格式, 预计 10 段左右即可; 也可以用树形图绘制, 一般不要少于 三层结构, 并且要在末级目录下列出相应的动画、图片和 视频等多种使用情况。通过这些多媒体的使用, 可以更好 的促进普通高校民俗体育网络教学平台的设计和实现。

4.2 我们要善于借助并应用合适的服务器。Tomcat 服 务器是一个免费的开放源代码的 Web 应用服务器, 也是一 个小型的轻量级的应用服务器, 适合在并发访问用户不是 很多的情况下以及一些中小型系统中使用, 是人们开发和 调试 JSP 程序的首选。在普通高校民俗体育网络教学平台 设计中, 我们可以充分应用它, 利用它运行时占用的系统 资源小, 并且扩展性比较好。其支持邮件服务和负载平衡 等开发应用系统常用的功能都比较好, 便于较好设计普通 高校民俗体育网络教学平台。比如: 可以在一台机器上配 置好 Apache 服务器, 通过可以利用它来响应对 HTML 页面 的访问请求。因为 Tomcat 部分是对 Apache 服务器的扩展, 尽管它是独立运行的。充分应用 Tomcat, 因为它在工作时 实际上是一个单独运行的进程, 并且可以和 Apache 可以相 独立开来。所以普通高校在对民俗体育网络教学平台进行 设计时, 在应用 Tomcat 时还可以不断的改进和完善它, 可 以给它加入新的功能, 使其更好的为普通高校民俗体育网 络教学平台的设计服务。

4.3 借助实时传输技术实现民俗体育示范动作的呈现。 我们可以在当前科技发展的水平下，充分采用实时控制协 
议 RTCP 和实时传输协议 RTP, 将它们用于 Internet 上, 针对多媒体数据流可以更好地在一对一传输或一对多传输 的情况下, 为我们提供时间信息, 并实现流同步。而且在 普通高校民俗体育网络教学平台设计中可以采用这些多媒 体技术，从而更好的实现民俗体育动作的清晰传授。

4.4 民俗体育网络课件可以扬长避短, 发挥优势。因 为传统的学校体育总是围绕着教师、课堂、训练对学生进 行教育, 使学生自主性的培养与发挥难以实现, 容易使学 生受到限制, 难以实现老师的因材施教和学生个性化学习。 普通高校在民俗体育网络教学平台的建立中要为体育教学 改革开辟新的途径, 提供一个庞大资料库, 汇集一些先进 的普通学校、图书馆、权威机构等各种有效的信息资源, 从而方便学习者学习体育、了解体育发展现状和当前体育 动态以及体育科技进步的最新知识。

\section{5. 结束语}

努力加强我国江西省普通高校民俗体育网络教学改 革, 有利于丢弃旧的体育教学观念, 引起江西省体育教学 和学习观念的变革, 并在全国普通高校民俗体育网络教学 起到更好的带头作用。同时, 要努力设计好普通高校民俗 体育网络教学的平台, 可以更好的向人们展示了一个广阔 的、崭新的体育世界, 并且为任何愿意获取体育信息和体 育知识的人提供了学习的机会; 更有利于使我国体育教学
活动逐渐地由知识传递型转变为重视对学生实践能力和创 造精神的培养, 进而实现由知识学习型向知识发展型转变, 为我国体育实现终身学习体制而奠定成熟的基础。

\section{参考文献(References)}

[1] Xie Hongchang, Yuan Jiang Nan. On the effective strategies of college sports learning website construction. Journal of Jilin Institute of Physical Education, 2008 (02).

[2] Wang Zhuzhu, Zhang Weiyuan. Colleges and universities in our country online teaching platforms and websites of the status quo analysis. Chinese distance education. 2005 (02)

[3] Personalized interactive network teaching platform [3] dragon Yinxiang.B/S model of the structure and design of. computer and modernization. 2005 (06)

[4] Shi Jiying, Zhang Lei, Qian Juxi. electrical and electronic teaching.2004 Chinese Journal of network teaching platform construction based on ASP and database technology (06)

[5] Zhang Li, Zhang Yan. From C/S to B/S, and then to the three layer (multilayer) structure -- on the development of architecture. Journal of Henan Normal University (NATURAL SCIENCE EDITION).2002 (03) 